\title{
Clear Cell Adenocarcinoma Arising from Adenofibroma in a Patient with Endometriosis of the Ovary
}

\author{
Inju Cho $\cdot$ Sung-Chul Lim \\ Department of Pathology, Chosun University \\ School of Medicine, Gwangju, Korea \\ Received: April 9, 2015 \\ Revised: July 23, 2015 \\ Accepted: August 7, 2015 \\ Corresponding Author \\ Sung-Chul Lim, MD, PhD \\ Department of Pathology, Chosun University \\ Hospital, 365 Pilmun-daero, Dong-gu, Gwangju \\ 61453, Korea \\ Tel: +82-62-230-6343 \\ Fax: +82-62-226-5860 \\ E-mail: sclim@chosun.ac.kr
}

\begin{abstract}
Ovarian clear cell adenocarcinomas (CCACs) are frequently associated with endometriosis and, less often with clear cell adenofibromas (CCAFs). We encountered a case of ovarian CCAC arising from benign and borderline adenofibromas of the clear cell and endometrioid types with endometriosis in a 53-year-old woman. Regions of the adenofibromas showed transformation to CCAC and regions of the endometriosis showed atypical endometriotic cysts. This case demonstrates that CCAC can arise from CCAF or endometriosis.
\end{abstract}

Key Words: Adenocarcinoma, clear cell; Adenofibroma; Endometriosis; Ovary
Histological and epidemiological analyses have demonstrated a close relationship between endometriosis and ovarian clear cell adenocarcinoma (CCAC). ${ }^{1-3}$ Clear cell adenofibroma (CCAF) is a major benign or borderline type of ovarian clear cell tumor. ${ }^{4}$ However, benign CCAF is extremely rare. ${ }^{5,6}$ One study demonstrated the coexistence of CCAF components (14 cases) in 21\% of surgically resected ovarian CCACs. Of these 14 CCAF (+) cases, CCAF with atypia were found adjacent to CCAF without atypia in 10, and adjacent to obvious CCACs in 13 cases. $^{7}$ Therefore, we can speculate that both CCAF and endometriosis may be precursors of CCAC. Several studies have demonstrated that there is a genetic linkage between $\mathrm{CCAF}$ and $\mathrm{CCAC}{ }^{8}$, as well as between endometriosis and CCAC.9-11

Here, we report a case of CCAC in a 53-year-old woman with both benign and borderline ovarian adenofibromas of the clear cell and endometrioid types, in addition to endometriosis.

\section{CASE REPORT}

A 53-year-old woman visited a local obstetrics and gynecology clinic for a routine examination. Ultrasonography demonstrated a right ovarian cystic mass. The patient was referred to the Department of Obstetrics and Gynecology at Chosun Uni- versity Hospital for further evaluation and treatment. The patient had a history of laparoscopic left salpingo-oophorectomy and adhesiolysis due to left ovarian teratoma and intestinal adhesion seven years earlier. She had also experienced an intracranial hemorrhage 3 years earlier, and had suffered from medication-controlled hypothyroidism for 5 years.

Abdominal computed tomography revealed a 5.5-cm cystic mass in her right ovary (Fig. 1A). The cyst was unilocular with fluid attenuation, and there was no contrast-enhancing solid mass. Laparoscopy findings revealed a fluid-containing cystic mass that had a smooth surface with focal hemorrhage (Fig. 1B). Laparoscopic right salpingo-oophorectomy and adhesiolysis was undertaken under the clinical impression of benign cystadenoma.

Gross findings revealed a previously collapsed cystic mass measuring $5 \mathrm{~cm} \times 4.5 \mathrm{~cm} \times 2.5 \mathrm{~cm}$. The mass showed a $2.3 \mathrm{~cm} \times$ $1.2 \mathrm{~cm}$ solid part on one side. Histologic examination revealed benign and borderline CCAFs, endometriosis, atypical endometriotic cysts, and CCAC. The solid portion consisted of benign and borderline CCAF, CCAC, and endometriosis in descending order of prevalence. The cystic portion was merged into the solid portion of CCAF and CCAC, and consisted of benign and atypical endometriotic cysts.

The benign CCAF constituted a major portion of the tumor 
and demonstrated a compact arrangement of variably sized tubulocystic structures in the cellular stroma with no nuclear atypia. Flattened indiscernible, flat cuboidal, and polygonal cells with relatively abundant cytoplasm and no nuclear atypia lined the mass (Fig. 2). The glands in the borderline CCAF showed a greater degree of crowding and variation in size and shape compared with the benign CCAF. Focal nuclear atypia was observed in the glandular linings. The glands were delineated with one to three layers of cells with abundant cytoplasm and mildly pleomorphic, hyperchromatic nuclei. In some areas, stratified epithelium showed tiny buds with atypical nuclei. However, the glands in many areas were lined with non-atypical flat to cuboidal epithelium (Fig. 2).

The CCAC had a tubulocystic pattern with hobnail, cuboidal or flat atypical cell lining, characterized by nuclear enlargement and hyperchromasia. The glands were separated by fibrous stro-
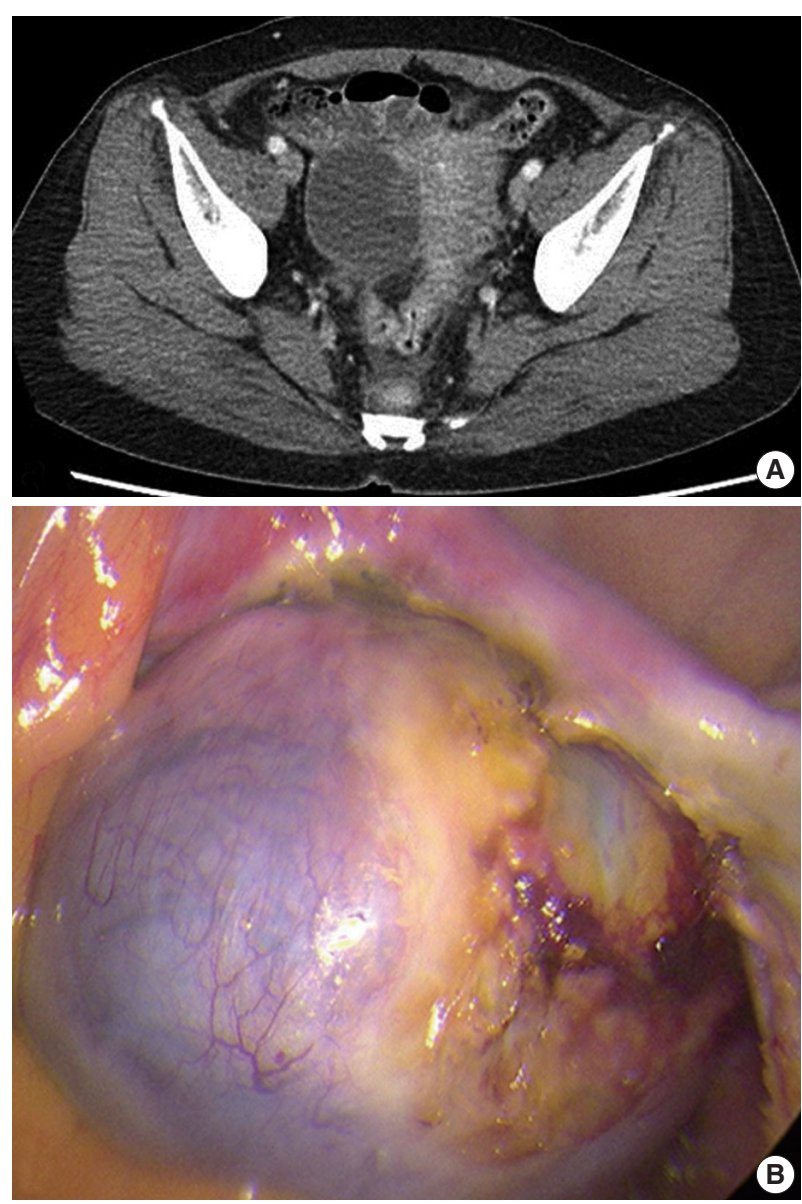

Fig. 1. Abdominal computed tomography (CT) and laparoscopic findings. (A) Abdominal computed tomography showing a $5.5-\mathrm{cm}$ cystic mass in the right ovary. (B) Laparoscopy findings reveal a fluid containing cystic mass with a smooth surface and focal hemorrhage. $\mathrm{ma}$, and foci of altered stromal responses were noted. There was crowding of tubules with a slightly haphazard arrangement in some areas. The borderline CCAF had microinvasion characterized by a few cells scattered haphazardly in the surrounding stroma. The borderline CCAF and CCAC were scattered, and a histologic continuum between borderline CCAF and CCAC was found within the same tumor (Fig. 2).

The benign endometriotic cysts had a predominantly cystic gross appearance. Typical endometriotic cysts showed minimally stratified tubal-type epithelium overlying the scant endometrial-type stroma. This endometriotic epithelium gradually showed greater nuclear atypia, demonstrating a multilayered epithelium with hyperchromatic, enlarged and irregular nuclei with prominent nucleoli as it merged with possible intraepithelial carcinomatous areas (Fig. 3).

The presence of benign and borderline CCAFs, borderline CCAF with microinvasion, CCAC, endometriosis, and benign and atypical endometriotic cysts within this single ovarian mass suggests a histologic continuum among endometriosis, CCAF and CCAC.

\section{DISCUSSION}

Sampson ${ }^{12}$ was the first to report malignant transformation of ovarian endometriosis. Endometrioid adenocarcinoma and CCAC are the most common types of malignancy arising from ovarian endometriosis. ${ }^{13}$ Ovarian endometriosis is therefore considered a precursor lesion to endometrioid adenocarcinoma and CCAC of the ovary. Sampson ${ }^{12}$ and Scott ${ }^{14}$ suggested the following criteria for determining whether a tumor has arisen from endometriosis: the presence of both malignant and benign endometrial tissue in the same ovary; cancer arising from ovarian endometriosis without other invasion; tissue resembling endometrial stroma surrounding characteristic epithelial glands; and a transition between benign endometriosis and malignant epithelium. In the present case, we found typical endometriosis, benign and atypical endometriotic cysts, and possible intraepithelial carcinomatous areas in the same ovary. CCAC arising in ovarian endometriosis or CCAF without other invasion was also found.

Malignant transformation of ovarian endometriosis occurs in more than $1 \%$ of the cases. ${ }^{12} \mathrm{CCAC}$ is the most common type of malignant transformation of ovarian endometriosis, and endometrioid adenocarcinoma is the second most common. ${ }^{13}$

Adenofibromas are also usually associated with ovarian endometriosis. ${ }^{5,15}$ In the present case, we identified benign and bor- 

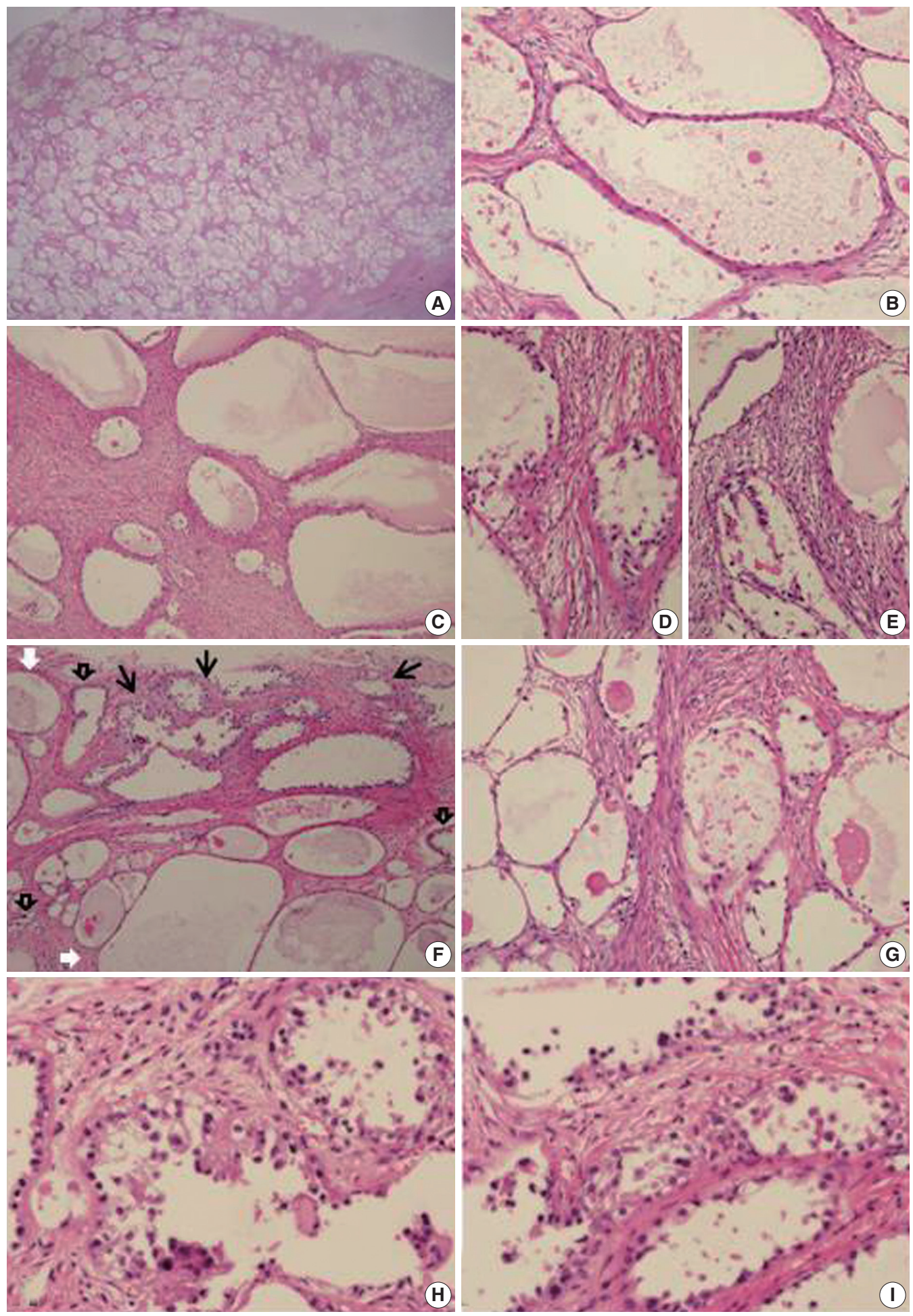

Fig. 2. Histopathologic findings of the solid part of the mass. (A) The compact arrangement of variably-sized tubulocystic structures in the stroma is consistent with adenofibroma. The cell lining consisted of flattened indiscernible cells or flat cuboidal cells (B) and polygonal cells with abundant clear cytoplasm (C). (D, E) In some areas, stratified epithelium shows tiny buds with atypical nuclei. (F) The transitional zone from benign (white arrows) to borderline (black open arrows) clear cell adenofibromas to clear cell adenocarcinoma (black arrows). (G) Higher magnification shows benign (left) and atypical (right) adenofibromas. (H, I) Area of clear cell adenocarcinoma shows a tubulocystic pattern with hobnail, cuboidal, or flat atypical lining cells characterized by nuclear enlargement and hyperchromasia, and foci of altered stromal responses. 

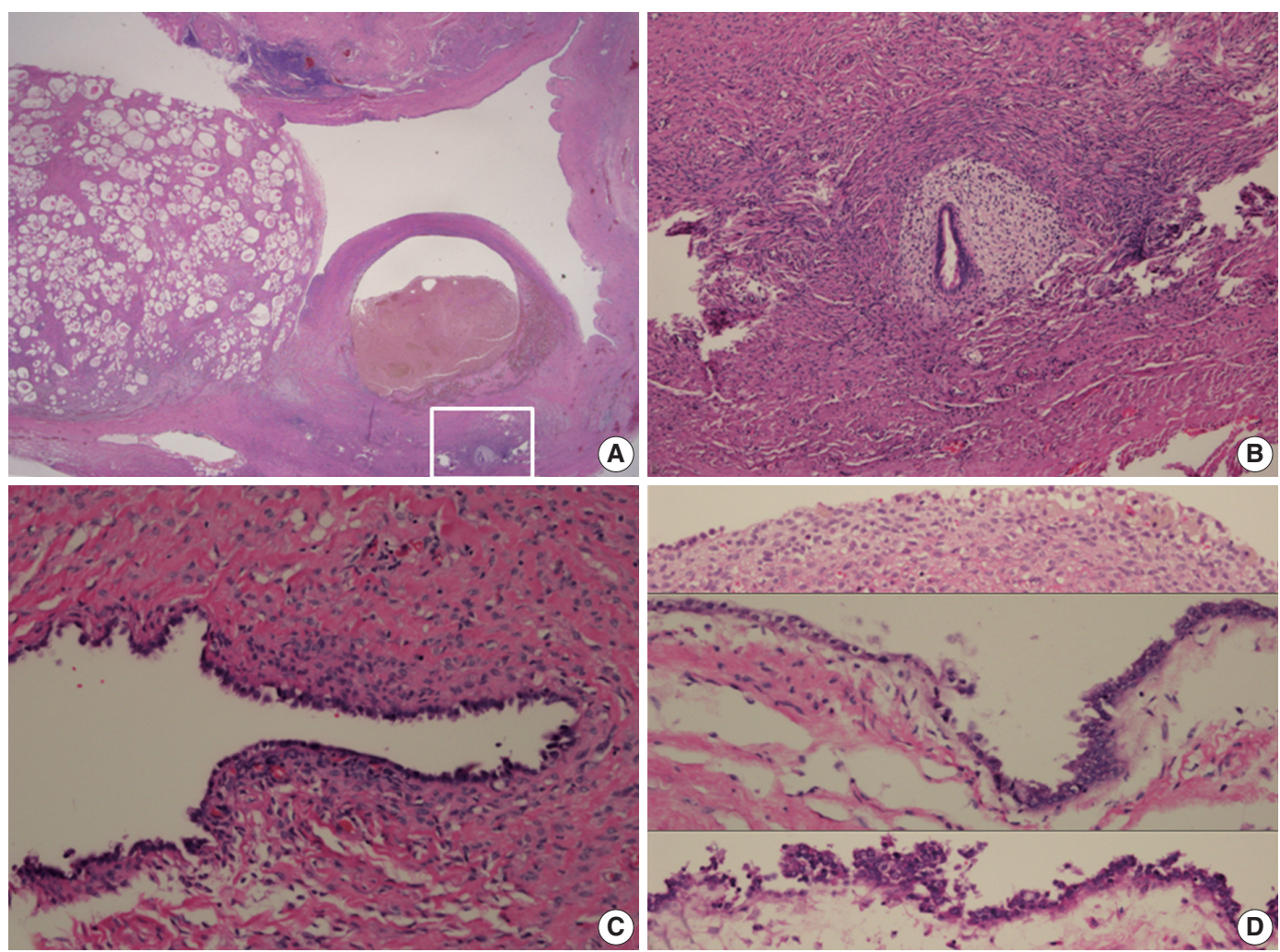

Fig. 3. Histopathologic findings of adenofibroma, endometriotic cyst, and endometriosis (A). Higher magnification of the inset shows endometriosis (B). (C) Endometriotic cyst with tubal-type epithelium overlying scant endometrial-type stroma. (D) Endometriotic cyst with simple cuboidal epithelium overlying endometrial stroma with hemosiderin pigmentation (upper) and gradual transition to nuclear atypia demonstrating stratification with hyperchromatic, enlarged, and irregular nuclei with prominent nucleoli (middle and lower).

derline adenofibromas, and the borderline adenofibromas demonstrated a transition to CCAC. Slow and progressive transformation of CCAC from benign to borderline to a microinvasive pattern has been suggested. ${ }^{5}$ In addition, patients with ovarian CCAF are younger than those with CCAC. ${ }^{5,6}$ This supports the suggestion of transformation of CCAC from benign to malignant tumors.

CCAC containing CCAFs are occasionally found. ${ }^{6,16,17}$ According to the classification of CCAC into groups with and without CCAF components, the CCAF (+) group showed a higher frequency of histologically low-grade tumors, a lower Ki-67 labeling index, less frequent endometriosis, and better patient prognosis than the CCAF (-) group.?

There are two types of ovarian carcinogenesis, type I and type II. Type I tumors are usually low grade and in low stage, behave in an indolent fashion, and develop slowly from precursor lesions. However, type II tumors are highly aggressive high grade tumors characterized by frequent TP53 mutations. They are not associated with the usual precursor lesions. CCAC is associated with precursor lesions such as endometriosis, adenofibromas, bor- derline/atypical adenofibromas and endometriotic cysts. It is also found in a low stage, showing a low frequency of TP53 mutations. However, CCAC is high grade and associated with poor prognosis when it is found in high stage. ${ }^{18}$ Zhao et al..$^{18}$ speculated that ovarian CCAC have two pathways. In one, epithelial atypia arises in an endometriotic cyst and then evolves into CCAC, and in the other, non-cystic endometriosis induces a fibromatous reaction resulting in the formation of an adenofibro$\mathrm{ma}$, which then develops into borderline adenofibroma and subsequently CCAC. Therefore, CCACs with or without adenofibromas are more closely related to type I tumors. Adenofibromatous and cystic types of CCAC appear to be derived from endometriosis. Adenofibromatous CCAC develops from noncystic endometriosis and is associated with an adenofibromatous background, while the cystic type of CCAC develops from an endometriotic cyst and is not associated with an adenofibromatous background. ${ }^{18}$ The two pathways may overlap in some cases. The present case showed benign and borderline CCAFs, borderline CCAF with microinvasion, CCAC, endometriosis, and benign and atypical endometriotic cysts. Thus, the two pathways 
overlapped in the present case. ARID1A mutation and loss of the corresponding protein, BAF250a, are common in CCAC. However, loss of BAF250a expression is significantly more common in CCAC with endometriosis than in cases with adenofibroma. ${ }^{19}$

There is a common genetic linkage between endometriosis and ovarian cancers such as CCAC, including losses of heterozygosity (LOHs) and alleles at the PTEN locus. ${ }^{9-11}$ Moreover, there is also a common genetic linkage between CCAF and ovarian CCAC, such as LOHs on 5q, 10q, and 22q. Therefore, possible alternative ovarian clear cell carcinogenic pathways are endometriosis to CCAC, CCAF to CCAC, or endometriosis to CCAF/ adenofibroma to CCAC.

The present case demonstrates that both CCAF and endometriosis should be regarded as precursors to CCAC.

\section{Conflicts of Interest}

No potential conflict of interest relevant to this article was reported.

\section{Acknowledgments}

This study was supported by research fund from Chosun University, 2014.

\section{REFERENCES}

1. Seidman JD, Russell P, Kurman RJ. Surface epithelial tumors of the ovary. In: Kurman RJ, editor. Blaustein's pathology of the female genital tract. 5th ed. New York: Springer-Verlag, 2001; 791-904.

2. Vercellini P, Parazzini F, Bolis G, et al. Endometriosis and ovarian cancer. Am J Obstet Gynecol 1993; 169: 181-2.

3. Fukunaga M, Nomura K, Ishikawa E, Ushigome S. Ovarian atypical endometriosis: its close association with malignant epithelial tumours. Histopathology 1997; 30: 249-55.

4. Tavassoli FA, Devilee P. World Health Organization classification of tumours of pathology and genetics of tumours of the breast and female genital organs. Lyon: IARC Press, 2003; 218-28.

5. Bell DA, Scully RE. Benign and borderline clear cell adenofibromas of the ovary. Cancer 1985; 56: 2922-31.

6. Roth LM, Langley FA, Fox H, Wheeler JE, Czernobilsky B. Ovarian clear cell adenofibromatous tumors: benign, of low malignant potential, and associated with invasive clear cell carcinoma. Cancer 1984; 53: 1156-63.
7. Yamamoto S, Tsuda H, Yoshikawa T, et al. Clear cell adenocarcinoma associated with clear cell adenofibromatous components: a subgroup of ovarian clear cell adenocarcinoma with distinct clinicopathologic characteristics. Am J Surg Pathol 2007; 31: 999-1006.

8. Yamamoto S, Tsuda H, Takano M, Hase K, Tamai S, Matsubara O. Clear-cell adenofibroma can be a clonal precursor for clear-cell adenocarcinoma of the ovary: a possible alternative ovarian clear-cell carcinogenic pathway. J Pathol 2008; 216: 103-10.

9. Jiang X, Morland SJ, Hitchcock A, Thomas EJ, Campbell IG. Allelotyping of endometriosis with adjacent ovarian carcinoma reveals evidence of a common lineage. Cancer Res 1998; 58: 1707-12.

10. Obata K, Hoshiai H. Common genetic changes between endometriosis and ovarian cancer. Gynecol Obstet Invest 2000; 50 Suppl 1: 39-43.

11. Sato N, Tsunoda H, Nishida M, et al. Loss of heterozygosity on 10q23.3 and mutation of the tumor suppressor gene PTEN in benign endometrial cyst of the ovary: possible sequence progression from benign endometrial cyst to endometrioid carcinoma and clear cell carcinoma of the ovary. Cancer Res 2000; 60: 7052-6.

12. Sampson JA. Endometrioid carcinoma of the ovary, arising in endometrial tissue in that organ. Arch Surg 1925; 10: 1-72.

13. Russell P. The pathological assessment of ovarian neoplasms. I: Introduction to the common 'epithelial' tumours and analysis of benign 'epithelial' tumours. Pathology 1979; 11: 5-26.

14. Scott RB. Malignant changes in endometriosis. Obstet Gynecol 1953; 2: 283-9.

15. Kao GF, Norris HJ. Unusual cystadenofibromas: endometrioid, mucinous, and clear cell types. Obstet Gynecol 1979; 54: 729-36.

16. Russell P, Merkur H. Proliferating ovarian "epithelial" tumours: a clinico-pathological analysis of 144 cases. Aust N Z J Obstet Gynaecol 1979; 19: 45-51.

17. Bell DA. Ovarian surface epithelial-stromal tumors. Hum Pathol 1991; 22: 750-62.

18. Zhao C, Wu LS, Barner R. Pathogenesis of ovarian clear cell adenofibroma, atypical proliferative (borderline) tumor, and carcinoma: clinicopathologic features of tumors with endometriosis or adenofibromatous components support two related pathways of tumor development. J Cancer 2011; 2: 94-106.

19. Nishikimi K, Kiyokawa T, Tate S, Iwamoto M, Shozu M. ARID1A expression in ovarian clear cell carcinoma with an adenofibromatous component. Histopathology 2015 Apr 23 [Epub]. http:/ / dx.doi.org/10.1111/his.12721. 